\title{
US health secretary quits over improper use of aircraft
}

An error was introduced in the editing of this news story $(B M J$ 2017;359:j4556, doi:10.1136/bmj.j4556). The 10th paragraph should say that it is the Association of American Physicians and Surgeons, not Tom Price himself, that opposes mandatory vaccination as "equivalent to human experimentation." 